\title{
ON THE GREEN'S FUNCTIONS OF ORDINARY DIFFERENTIAL SYSTEMS
}

\section{KENNETH S. MILLER AND MENAHEM M. SCHIFFER}

1. Introduction. In the theory of partial differential equations, variational formulas for the Green's function are well known. The first formula of this type was derived by Hadamard in the case of Laplace's equation (see, for example, $[1, \mathrm{pp} .303-305]) .{ }^{1}$ Let us consider the first boundary value problem in potential theory which consists in finding a function $u(x, y, z)$ satisfying $\partial^{2} u / \partial x^{2}+\partial^{2} u / \partial y^{2}$ $+\partial^{2} u / \partial z^{2}=0$ in a three-dimensional finite domain $V$ bounded by a smooth surface $S$ and taking on certain assigned values on this surface. This problem can always be solved by means of Green's function $g(P, Q)$ depending on the two points $P$ and $Q$ in $V . g$ is harmonic in $V$ in either argument point, except for $P=Q$ where it becomes infinite like $1 / \overline{P Q}$; it vanishes if either argument point lies on the boundary $S$ of $V$.

Suppose now that the region $V$ is slightly changed to $V^{\prime}$ whose boundary $S^{\prime}$ is obtained from $S$ by shifting every boundary point by an amount $\delta n$ along the interior normal. Let $g^{\prime}(P, Q)$ be Green's function belonging to $V^{\prime}$ and denote $\delta g(P, Q)$ by

$$
\delta g(P, Q)=\frac{1}{4 \pi} \iint_{S} \frac{\partial}{\partial n_{A}} g(A, P) \frac{\partial}{\partial n_{A}} g(A, Q) \delta n_{A} d s_{A} ;
$$

then $g^{\prime}(P, Q)-g(P, Q)=\delta g(P, Q)+o(\delta n) . \delta g(P, Q)$ is called the variation of Green's function. It plays the same role in the theory of the function $g(P, Q)$ which depends on the surface $S$ as the differential plays in the theory of a function of finitely many variables.

Interesting problems and methods of functional analysis are connected with Hadamard's formula for $\delta g$. In this paper, analogous but simpler variational formulas will be given for the Green's function of an ordinary differential system. Since the boundary of the domain considered now consists only of the two end points of the interval, the dependence of Green's function on the domain can be treated by ordinary calculus instead of functional analysis. Nevertheless, a great formal similarity of the results prevails. We shall derive, for example, in $\$ 4.2$ the formula

Presented to the International Congress of Mathematicians, September 6, 1950; received by the editors October 3, 1951 .

${ }^{1}$ Numbers in brackets refer to the references at the end of this paper. 


$$
\delta G(x, \xi)=\frac{\partial^{r}}{\partial x^{r}} G(b, \xi) \frac{\partial^{r}}{\partial \xi^{r}} G(x, b) p_{0}(b) \delta b
$$

which stands in striking analogy to Hadamard's formula.

We start this paper by collecting a few results, definitions, and conventions that will be used throughout. Our first result is to show how a fundamental system of solutions of a linear differential operator can be obtained from the Green's function of the system. This shows that once the Green's function of the system is known, all boundary value problems can easily be solved. In $\$ 4$, the variational formulas are considered, i.e., how the Green's function varies as interval, operator, and boundary conditions are changed. Both finite comparison and infinitesimal variational formulas are derived.

2. Preliminary remarks. Our main concern will be an ordinary differential operator of order $n$ which we shall write as

$$
L=p_{0}(x) \frac{d^{n}}{d x^{n}}+p_{1}(x) \frac{d^{n-1}}{d x^{n-1}}+\cdots+p_{n}(x) .
$$

The coefficients will be assumed to be continuous in some closed finite interval $I$ of the $x$-axis, and $p_{0}(x)>0$ in $I$. Frequently it will be convenient to consider the formal or Lagrange adjoint of $L$. This we denote by $L^{+}$,

$$
L^{+}=p_{0}^{*}(x) \frac{d^{n}}{d x^{n}}+p_{1}^{*}(x) \frac{d^{n-1}}{d x^{n-1}}+\cdots+p_{n}^{*}(x) .
$$

With the formal differential operator $L$ we must often associate boundary conditions. These will be the most general two-point boundary conditions of the form

$$
U_{\alpha}(u)=\sum_{i=1}^{n} c_{\alpha, i} u^{(i-1)}(a)+\sum_{i=1}^{n} c_{\alpha, n+i} u^{(i-1)}(b), \quad i=1,2, \cdots, n .
$$

Here $a<b$ and the closed interval $[a, b]$ is a subinterval of $I$. It will always be assumed that the completely homogeneous differential system

$$
L u=0, \quad U_{\alpha}(u)=0, \quad \alpha=1,2, \cdots, n,
$$

is incompatible; that is, the only solution of $(4)$ is $u(x) \equiv 0$. Sometimes it will be convenient to write

$$
V_{\alpha}(u)=\sum_{i=1}^{n} c_{\alpha, i} u^{(i-1)}(a), \quad Z_{\alpha}(u)=\sum_{i=1}^{n} c_{\alpha, n+i} u^{(i-1)}(b) .
$$


Then $U_{\alpha}(u)=V_{\alpha}(u)+Z_{\alpha}(u)$. In a similar way the adjoint boundary conditions will be denoted by

$$
U_{\alpha}^{*}(u)=\sum_{i=1}^{n}\left[c_{\alpha, i}^{*} u^{(i-1)}(a)+c_{\alpha, n+i}^{*} u^{(i-1)}(b)\right], \quad \alpha=1,2, \cdots, n .
$$

For every incompatible differential system of the form (4) there exists a Green's function $G(x, \xi)$. Sometimes it will be written $G(x, \xi, a, b)$ or $G\left(x, \xi, c_{\alpha i}\right)$ to indicate that it also depends on the interval or on the boundary conditions. When no confusion will arise the arguments in the third and fourth places will be suppressed.

A fundamental tool in the investigations we are about to undertake is Green's formula. Associated with this formula is the so-called Lagrange bilinear concomitant. We shall say a few words about this form. Let $u(x)$ and $v(x)$ be two functions of $x$ whose $(n-1)$ st derivatives exist in $I$. Then the expression $[2$, p. 123]

$$
\begin{aligned}
P[u(x), v(x)] \equiv & u^{(n-1)}(x) p_{0}(x) v(x)-u^{(n-2)}(x)\left[p_{0}(x) v(x)\right]^{\prime}+\cdots \\
& +(-1)^{n-1} u(x)\left[p_{0}(x) v(x)\right](n-1)+\cdots \\
& +u^{\prime}(x) p_{n-2}(x) v(x)-u(x)\left[p_{n-2}(x) v(x)\right]^{\prime} \\
& +u(x) p_{n-1}(x) v(x)
\end{aligned}
$$

is well defined. Now suppose $u(x)$ and $v(x)$ have $n$ derivatives in $I$. In this case we know $v(x) L u(x)-u(x) L^{+} v(x)=d P[u(x), v(x)] / d x$ from which follows

$$
\begin{aligned}
& \int_{a}^{b}\left\{v(x) L u(x)-u(x) L^{+} v(x)\right\} d x \\
&=P[u(b), v(b)]-P[u(a), v(a)] \equiv \pi[u, v] .
\end{aligned}
$$

These apparently trivial remarks will be used many times. For, if Green's formula is applied with $u$ or $v$ (or both) Green's functions, these functions will have a discontinuity in their $(n-1)$ st derivatives. Hence it is necessary to investigate carefully the bilinear form $P$.

The expression $\pi[u, v]$ may also be written as $[3$, p. 30] $\pi[u, v]$ $=\sum_{i=1}^{2 n} U_{i}(u) U_{2 n+1-i}^{*}(v)$ where $U_{1}, U_{2}, \cdots, U_{n}$ have the meaning ascribed to them by (3) and $U_{n+1}, \cdots, U_{2 n}$ are any other $n$ linear boundary conditions so chosen that the rank of the matrix of the coefficients $\left\|c_{\alpha i}\right\|, \alpha=1,2, \cdots, 2 n ; i=1,2, \cdots, 2 n$, is precisely $2 n$. The $U_{\alpha}^{*}$ will then be determined; and it is shown in the above quoted reference that the $U_{\alpha}^{*}, \alpha=1,2, \cdots, n$, have the property of being the boundary conditions associated with the adjoint equation. [See (5).] 
We conclude this section with a few remark on notation. It will sometimes be convenient to use the symbolism $\partial^{\beta} G(x, t, a, b) / \partial 2^{\beta}$ in place of $\partial^{\beta} G(x, t, a, b) / \partial t^{\beta}$ or again $G_{1}(x, t, a, b)$ in place of $\partial G(x, t, a, b) / \partial x$. The differential expression $L_{\zeta}$ is to mean that $L$ operates on the variable $\zeta$. Similarly $\zeta U$ is to mean that $U$ is to operate on functions of $\zeta$. In this notation, (4) could have been written as $L_{x} u=0,{ }^{x} U_{\alpha}(u)=0$. When the subscript on the $L$ or the pre-superscript on the $U$ is omitted, it is understood that $L$ and $U$ operate on the variable $x$.

3. Representation of a fundamental system in terms of the Green's function. It is well known that if we have a fundamental system of solutions of the linear differential equation $L u=0$, a Green's function can be constructed for the system of (4) where the $U_{\alpha}(u)$ can be any two-point boundary conditions subject only to the restriction that the completely homogeneous system be incompatible. One would surmise that the converse is true. In Theorem 1 below we obtain this result.

TheOREM 1. Hypothesis. Let $L u=0, U_{\alpha}(u)=0, \alpha=1,2, \cdots, n$, be the linear differential system defined by (4) and let $L^{+} u=0, U_{\alpha}^{*}(u)=0$ be the adjoint system. Let $G(x, \xi)$ be the Green's function for the system of (4).

Conclusion. Among the $2 n$ functions

$$
\psi_{\beta}(x)=\left.\frac{\partial^{\beta-1}}{\partial \xi^{\beta-1}} G(x, \xi)\right|_{\xi=a}, \quad \psi_{n+\beta}(x)=\left.\frac{\partial^{\beta-1}}{\partial \xi^{\beta-1}} G(x, \xi)\right|_{\xi=b},
$$

$\beta=1,2, \cdots, n$, there exist precisely $n$ linearly independent solutions of the equation $L u=0$. If $\left\|c_{i, j_{\beta}}^{*}\right\|, i=1,2, \cdots, n, \beta=1,2, \cdots, n$, is a nonsingular sub-matrix of the matrix $\left\|c_{i, j}^{*}\right\|, i=1,2, \cdots, n, j$ $=1,2, \cdots, 2 n$, of the $n$ linear forms $U_{\alpha}^{*}, \alpha=1,2, \cdots, n$, then $\psi_{j_{n+\beta}}(x), \beta=1,2, \cdots, n$, may be chosen as such a set of linearly independent solutions of $L u=0$.

Proof. It is evident that each $\psi_{\beta}(x), \beta=1,2, \cdots, 2 n$, is a solution of $L u=0$, and hence that there are at most $n$ linearly independent functions among the $2 n$ functions $\psi_{\beta}(x)$. We shall prove that there are at least $n$.

Consider Green's identity ( 7$)$ where $u=\phi(x)$ is any solution of $L u=0$ and $v=G^{*}(x, \xi)$ is the Green's function for the adjoint system. Then

$$
0=\int_{a}^{b}\left\{G^{*}(x, \xi) L \phi(x)-\phi(x) L^{+} G^{*}(x, \xi)\right\} d x .
$$


Expanding and simplifying we obtain $\phi(\xi)=-\pi\left[\phi, G^{*}\right]$. But the expression $\pi\left[\phi, G^{*}\right]$ is a linear combination of the functions $\psi_{\beta}(\xi)$ with constant coefficients. Hence

$$
\phi(x)=\sum_{i=1}^{2 n} A_{i} \psi_{i}(x), \quad A_{i}=\text { constant }
$$

Equation (8) is valid for any function $\phi(x)$ such that $L \phi=0$. Hence if $\left\{\phi_{1}(x), \phi_{2}(x), \cdots, \phi_{n}(x)\right\}$ form a fundamental system for $L u=0$, we may write $\phi_{\alpha}(x)=\sum_{i=1}^{2 n} A_{\alpha i} \psi_{i}(x), \alpha=1,2, \cdots, n$.

We shall now determine a particular subset of $n$ functions of the $2 n$ functions $\psi_{\beta}(x)$ which have the property enounced in the theorem. Consider the matrix $\left\|c_{i, j}^{*}\right\|, i=1,2, \cdots, n, j=1,2, \cdots, 2 n$, of the coefficients of the linear forms $U_{\alpha}^{*}, \alpha=1,2, \cdots, n$. Since the $U_{\alpha}^{*}$ are linearly independent, the rank of this matrix is $n$. Let $\left\|c_{i, j}^{*}\right\|, i, \beta$ $=1,2, \cdots, n$, be any (if there are more than one) of these nonsingular matrices. We may then solve the $n$ equations ${ }^{x} U_{\alpha}^{*}\left[G^{*}(x, \xi)\right]$ $=0, \alpha=1,2, \cdots, n$, for the $\psi_{j_{\beta}}(\xi), \beta=1,2, \cdots, n$, in terms of the $\psi_{j_{n+\beta}}(\xi)$. This means that, since the $\psi_{j_{\beta}}$ are linearly dependent on the $\psi_{j_{n+\beta}}$ and there exist exactly $n$ linearly independent functions among the $2 n$ functions $\psi_{\beta}$, the $\psi_{j_{n+\beta}}(x), \beta=1,2, \cdots, n$, are linearly independent.

4. Finite comparison and infinitesimal variational formulas. In this section we shall be concerned with deriving formulas for the Green's function of a differential system which differs slightly (in some respect) from another differential system. First we shall obtain finite comparison formulas. By this we mean the following. Let the Green's function $G(x, \xi)$ of a given differential system depend on a parameter $\lambda$. Then we write $G(x, \xi)=G(x, \xi, \lambda)$ to exhibit this dependence explicitly. Let $G^{\prime}(x, \xi)$ be the Green's function for the system when $\lambda$ has been changed to $\lambda^{\prime}$. We write $G^{\prime}(x, \xi)=G^{\prime}\left(x, \xi, \lambda^{\prime}\right)$. By definition

$$
\Delta G(x, \xi)=G^{\prime}\left(x, \xi, \lambda^{\prime}\right)-G(x, \xi, \lambda)
$$

which also equals $\Delta G(x, \xi)=(\partial G(x, \xi, \lambda) / \partial \lambda) \Delta \lambda+O\left(\Delta \lambda^{2}\right)$. Let $\delta G(x, \xi)$ be the principal part of this expression. That is,

$$
\delta G(x, \xi)=\frac{\partial}{\partial \lambda} G(x, \xi, \lambda) \delta \lambda
$$

where the finite increment $\Delta \lambda$ of the independent variable has been identified with $\delta \lambda$. Equation (9) is called a finite comparison formula and (10) an infinitesimal variational formula. In general we obtain 
the infinitesimal case from the finite case, and by so doing simplify the formulas.

4.1. Variation of the interval. Consider the system of (4). Let $G(x, \xi, a, b)$ be its Green's function where we have brought into prominence the fact that $G$ depends on the end points of the interval. The system

$$
\begin{gathered}
L u=0, \\
U_{\alpha}\left(u^{\prime}\right)=\sum_{i=1}^{n} c_{\alpha, i} u^{(i-1)}\left(a^{\prime}\right)+c_{\alpha, n+i} u^{(i-1)}\left(b^{\prime}\right), \quad \alpha=1,2, \cdots, n,
\end{gathered}
$$

is identical with that of (4) except that $a$ has been replaced by $a^{\prime}$ and $b$ by $b^{\prime}$. Let $G^{\prime}\left(x, \xi, a^{\prime}, b^{\prime}\right)$ be the Green's function for the system of (11). Then in accordance with the principle described above

$$
\begin{aligned}
\Delta G(x, \xi) & =G^{\prime}\left(x, \xi, a^{\prime}, b^{\prime}\right)-G(x, \xi, a, b) \\
& =\frac{\partial}{\partial a} G(x, \xi, a, b) \Delta a+\frac{\partial}{\partial b} G(x, \xi, a, b) \Delta b+O\left(\Delta a^{2}, \Delta b^{2}\right) .
\end{aligned}
$$

Let $\delta G$ be the principal part of $\Delta G . \delta G(x, \xi)=(\partial G(x, \xi, a, b) / \partial a) \delta a$ $+(\partial G(x, \xi, a, b) / \partial b) \delta b$. We are now in a position to state precisely the theorem regarding the variation of the Green's function due to a change in domain.

Theorem 2. Hypothesis. Let the system $L u=0, U_{\alpha}(u)=0$ and $L u=0$, $U_{\alpha}\left(u^{\prime}\right)=0, \alpha=1,2, \cdots, n$, be as defined by (4) and (11). Let $G(x, \xi)$ and $G^{\prime}(x, \xi)$ be their corresponding Green's functions.

Conclusion. $\Delta G(x, \xi)=-\pi\left[G^{\prime}, G\right]$,

$$
\delta G(x, \xi)=\sum_{i=1}^{n} \zeta U_{2 n+1-i}^{*}[G(x, \zeta)]\left\{\zeta V_{i}\left[G_{1}(\zeta, \xi)\right] \delta a+\zeta Z_{i}\left[G_{1}(\zeta, \xi)\right] \delta b\right\} .
$$

Proof. Consider Green's identity (7) with $u=G^{\prime}(\zeta, \xi)$ and $v$ $=G(x, \zeta)$. Then $J(x, \xi)=\int_{a}^{b}\left\{G(x, \zeta) L_{\zeta} G^{\prime}(\zeta, \xi)-G^{\prime}(\zeta, \xi) L_{\zeta}^{+} G(x, \zeta)\right\} d \zeta$ $=0$ since $G(x, \zeta)=G^{*}(\zeta, x)$. Furthermore we deduce $0=J(x, \xi)$ $=G^{\prime}(x, \xi)-G(x, \xi)+\pi\left[G^{\prime}, G\right]$. Now we have seen that $\pi\left[G^{\prime}, G\right]$ may be written

$$
\pi\left[G^{\prime}, G\right]=\sum_{i=1}^{2 n} \zeta U_{i}\left[G^{\prime}(\zeta, \xi)\right] \zeta U_{2 n+1-i}^{*}[G(x, \zeta)] .
$$

We would like to eliminate $G^{\prime}$ from the right-hand side of this expression. In other words, we shall endeavor to get a formula for $\delta G$ in terms of $G, U, U^{*}, \delta a, \delta b$ alone. Towards this end consider the fol- 
lowing identity: ${ }^{\zeta} U_{\alpha}[G(\zeta, \xi, a, b)] \equiv 0, \alpha=1,2, \cdots, n$. [Since $G$ satisfies the boundary conditions, $U_{\alpha}(G)=0, \alpha=1,2, \cdots, n$.] In more extended form this identity becomes

$$
0=\sum_{i=1}^{n} c_{\alpha, i} \frac{\partial^{i-1}}{\partial 1^{i-1}} G(a, \xi, a, b)+\sum_{i=1}^{n} c_{\alpha, n+i} \frac{\partial^{i-1}}{\partial 1^{i-1}} G(b, \xi, a, b) .
$$

Differentiating the above identity with respect to $a$ and $b$ respectively we obtain $0 \equiv 5 V_{\alpha}\left[G_{1}(\zeta, \xi, a, b)\right]+{ }^{5} U_{\alpha}\left[G_{3}(\zeta, \xi, a, b)\right]$ and 0 $\equiv^{5} U_{\alpha}\left[G_{4}(\zeta, \xi, a, b)\right]+{ }^{\zeta} Z_{\alpha}\left[G_{1}(\zeta, \xi, a, b)\right]$ respectively. Hence $\zeta U_{\alpha}\left\{G_{3}(\zeta, \quad \xi, \quad a, \quad b) \delta a+G_{4}(\zeta, \quad \xi, \quad a, \quad b) \delta b\right\}=-\zeta V_{\alpha}\left[G_{1}(\zeta, \quad \xi)\right] \delta a$ $-\zeta Z_{\alpha}\left[G_{1}(\zeta, \xi)\right] \delta b$. Now $\delta G(x, \xi)=G_{3}(x, \xi, a, b) \delta a+G_{4}(x, \xi, a, b) \delta b$. Therefore $5 U_{\alpha}[\delta G(\zeta, \xi)]=-{ }^{\zeta} V_{\alpha}\left[G_{1}(\zeta, \xi)\right] \delta a-\zeta Z_{\alpha}\left[G_{1}(\zeta, \xi)\right] \delta b$. But ${ }^{\zeta} U_{\alpha}[G(\zeta, \xi)]=0, \alpha=1,2, \cdots, n$. Therefore, to first order variation ${ }^{\zeta} U_{\alpha}\left[G^{\prime}(\zeta, \xi)\right]=-\zeta V_{\alpha}\left[G_{1}(\zeta, \xi)\right] \delta a-\zeta Z_{\alpha}\left[G_{1}(\zeta, \xi)\right] \delta b, \alpha=1,2, \cdots, n$. Substituting this result in (12) and noting that ${ }^{5} U_{\beta}^{*}[G(x, \zeta)]$ $=\zeta U_{\beta}^{*}\left[G^{*}(\zeta, x)\right]=0$ for $\beta=1,2, \cdots, n$, we obtain the formula for $\delta G(x, \xi)$ which appears in the statement of the theorem.

4.2. An example. We consider an application of the above theorem to a special differential system.

Theorem 3. Hypothesis. Let the differential system be as in (4) where $L$ is of even order $n=2 r$ and where the boundary conditions assume the special form $U_{\alpha}(u)=u^{(\alpha-1)}(a), U_{r+\alpha}(u)=u^{(\alpha-1)}(b), \alpha=1,2, \cdots, r$.

Conclusion.

$$
\begin{aligned}
\delta G(x, \xi)=(-1)^{r}\left[\frac{\partial^{r}}{\partial x^{r}} G(b, \xi)\right. & \frac{\partial^{r}}{\partial \xi^{r}} G(x, b) p_{0}(b) \delta b \\
& \left.-\frac{\partial^{r}}{\partial x^{r}} G(a, \xi) \frac{\partial^{r}}{\partial \xi^{r}} G(x, a) p_{0}(a) \delta a\right] .
\end{aligned}
$$

Proof. Since $U_{\alpha}(u)=u^{(\alpha-1)}(a)$ and $U_{r+\alpha}(u)=u^{(\alpha-1)}(b)$, we may take $U_{2 r+\alpha}(u)=u^{(r+\alpha-1)}(a)$ and $U_{3 r+\alpha}\left(u^{\prime}\right)=u^{(r+\alpha-1)}(b)$ for $\alpha=1,2, \cdots$, $r$. From the bilinear form $P[u(x), v(x)]$ we readily deduce the adjoint boundary conditions $U_{i}^{*}(v), i=1,2, \cdots, 4 r$. Since ${ }^{\zeta} U_{\alpha}[G(\zeta, x)]$ $=0, \alpha=1,2, \cdots, n$, we see that $\zeta U_{\alpha}\left[G_{1}(\zeta, x)\right]=0, \alpha=1,2, \cdots$, $r-1, r+1, \cdots, n-1$. Now $5 U_{\alpha}\left[G_{1}(\zeta, x)\right] \equiv V_{\alpha}\left[G_{1}(\zeta, x)\right], \alpha=1,2$, $\cdots, r, 2 r+1, \cdots, 3 r$, and $\zeta U_{\alpha}\left[G_{1}(\zeta, x)\right] \equiv Z_{\alpha}\left[G_{1}(\zeta, x)\right], \alpha=r+1$, $\cdots, 2 r, 3 r+1, \cdots, 4 r$. We conclude therefore that

$$
\begin{aligned}
{ }^{\zeta} U_{3 r+1}^{*}[G(x, \zeta)] & =\zeta U_{3 r+1}^{*}\left[G^{*}(\zeta, x)\right]=\zeta V_{3 r+1}^{*}\left[G^{*}(\zeta, x)\right] \\
& =(-1)^{r} p_{0}(a) \frac{\partial^{r}}{\partial 1^{r}} G^{*}(a, x),
\end{aligned}
$$




$$
\begin{aligned}
\zeta U_{n+1}^{*}[G(x, \zeta)] & =\zeta Z_{2 r+1}\left[G^{*}(\zeta, x)\right]=(-1)^{r} p_{0}(b) \frac{\partial^{r}}{\partial 1^{r}} G^{*}(b, x), \\
\zeta V_{r}\left[G_{1}(\zeta, \xi)\right] & =\zeta U_{r}\left[G_{1}(\zeta, \xi)\right]=\frac{\partial^{r-1}}{\partial 1^{r-1}} G_{1}(a, \xi)=\frac{\partial^{r}}{\partial 1^{r}} G(a, \xi), \\
\zeta Z_{n}\left[G_{1}(\zeta, \xi)\right] & =\zeta U_{n}\left[G_{1}(\zeta, \xi)\right]=\frac{\partial^{r-1}}{\partial 1^{r-1}} G_{1}(b, \xi)=\frac{\partial^{r}}{\partial 1^{r}} G(b, \xi) .
\end{aligned}
$$

From these relations we deduce the formula enounced in Theorem 3 .

4.3. Variation of the operator. Again we consider the system of (4). Here $L$ is as in (1) and we define $L^{\prime}$ as

$$
L^{\prime}=p_{0}^{\prime}(x) \frac{d^{n}}{d x^{n}}+p_{1}^{\prime}(x) \frac{d^{n-1}}{d x^{n-1}}+\cdots+p_{n}^{\prime}(x) .
$$

[The primes on the $p_{i}(x)$ are not derivatives.] Let

$$
\begin{aligned}
\Delta L=L^{\prime}-L & =\Delta p_{0}(x) \frac{d^{n}}{d x^{n}}+\Delta p_{1}(x) \frac{d^{n-1}}{d x^{n-1}}+\cdots+\Delta p_{n}(x) . \\
& =\sum_{i=0}^{n} \frac{\partial L}{\partial p_{i}} \Delta p_{i}=\delta L=\sum_{i=0}^{n} \frac{\partial L}{\partial p_{i}} \delta p_{i} .
\end{aligned}
$$

We shall assume that $\Delta p_{i}(x) \in C^{n}$ in $[a, b]$ and that $d^{\alpha} \Delta p_{i}(x) / d x^{\alpha}$ is small for $i, \alpha=0,1, \cdots, n$. That is $O\left(d^{\alpha} \Delta p_{i}(x) / d x^{\alpha}\right)=O\left(\Delta p_{i}(x)\right)$. If $G\left(x, \xi, p_{i}\right)$ is the Green's function associated with the differential system $L^{\prime} u=0, U_{\alpha}(u)=0$, we have $\Delta G(x, \xi)=G^{\prime}\left(x, \xi, p_{i}^{\prime}\right)-G\left(x, \xi, p_{i}\right)$ $=\sum_{i=0}^{n}\left(\partial G\left(x, \xi, p_{i}\right) / \partial p_{i}\right) \Delta p_{i}+O\left(\Delta p_{i}^{2}\right)$ and as before $\delta G(x, \xi)$ $=\sum_{i=0}^{n}\left(\partial G\left(x, \xi, p_{i}\right) / \partial p_{i}\right) \delta p_{i}$.

Theorem 4. Hypothesis. Let the systems $L u=0, U_{\alpha}(u)=0$ and $L^{\prime} u$ $=0, U_{\alpha}(u)=0$ be as defined by (4) and (14). Let $G(x, \xi)$ and $G^{\prime}(x, \xi)$ be the corresponding Green's functions. Let $\Delta G$ and $\delta G$ be as defined above.

Conclusion.

$$
\begin{aligned}
\Delta G(x, \xi) & =-\int_{a}^{b} G(x, \zeta) \Delta L_{\zeta} G^{\prime}(\zeta, \xi) d \zeta \\
\delta G(x, \xi) & =-\int_{a}^{b} G(x, \zeta) \delta L_{\zeta} G(\zeta, \xi) d \zeta
\end{aligned}
$$

Proof. The proof follows as in the previous theorems by use of Green's formula applied to the Green's functions $G$ and $G^{\prime}$.

4.4. Variation of the boundary conditions. Let $U_{\alpha}(u), \alpha=1,2, \cdots$, 
$n$, be a given set of boundary conditions as defined by (3). Let $U_{\alpha}^{\prime}(u)$ be the perturbed boundary conditions.

$$
U_{\alpha}^{\prime}(u)=\sum_{i=1}^{n} c_{\alpha, i}^{\prime} u^{(i-1)}(a)+\sum_{i=1}^{n} c_{\alpha, n+i}^{\prime} u^{(i-1)}(b), \quad \alpha=1,2, \cdots, n .
$$

Following the pattern established in $\$ \S 4.1$ and 4.3 we define $\Delta U_{\alpha}(u)$ $=\delta U_{\alpha}(u), \alpha=1,2, \cdots, n$, in the expected fashion.

Let $G\left(x, \xi, c_{\alpha i}\right)$ be the Green's function associated with $L u=0$, $U_{\alpha}(u)=0$ and $G^{\prime}\left(x, \xi, c_{\alpha f}^{\prime}\right)$ be the Green's function associated with the system $L u=0, \quad U_{\alpha}^{\prime}(u)=0, \quad \alpha=1,2, \cdots, n$. Then $\Delta G(x, \xi)$ $=G^{\prime}\left(x, \xi, c_{\alpha i}^{\prime}\right)-G\left(x, \xi, c_{\alpha i}\right)$ and $\delta G(x, \xi)=\sum_{\alpha=1}^{n} \sum_{i=1}^{2 n}\left(\partial G / \partial c_{\alpha i}\right) \delta c_{\alpha i}$.

TheOREM 5. Hypothesis. Let $L u=0, U_{\alpha}(u)=0$ be the differential system as defined by (4) and let $L u=0, U_{\alpha}^{\prime}(u)=0$ be the differential system as defined by (16). Let $G(x, \xi)$ and $G^{\prime}(x, \xi)$ be their respective Green's functions.

Conclusion.

$$
\begin{aligned}
& \Delta G(x, \xi)=\sum_{i=1}^{n} \varsigma \Delta U_{i}\left[G^{\prime}(\zeta, \xi)\right] \zeta U_{2 n+1-i}[G(x, \zeta)], \\
& \delta G(x, \xi)=\sum_{i=1}^{n} \varsigma U_{2 n+1-i}^{*}[G(x, \zeta)]^{\zeta} \delta U_{i}[G(x, \xi)] .
\end{aligned}
$$

Proof. The proof follows by the same methods used in earlier theorems.

\section{REFERENCES}

1. J. Hadamard, Leçons sur le calcul des variations, vol. I, Paris, Hermann, 1910.

2. E. L. Ince, Ordinary differential equations, London, Longmans, Green, 1927.

3. M. Bôcher, Leçons sur les méthodes de Sturm, Paris, Gauthier-Villars, 1917

NeW YoRK UNIVERSITY AND

Princeton University 